\title{
Induced Representations and Free Field Equations
}

\author{
G. Grensing \\ Physics Department University of Kiel, Kiel, Germany \\ (Z. Naturforsch. 30 a, $583-593$ [1975] ; received November 2, 1974)
}

\begin{abstract}
We give a simple and genuine method for the derivation of free field equations. Within our approach, the field equations represent necessary and sufficient conditions for the field representation to be a unitary and irreducible representation of a covering group of the full Poincaré group. The method is applied to particles with spin less than or equal to one.
\end{abstract}

\section{Introduction}

If a further paper is added to this intensively cultivated territory, one is forced to give reasons: For our taste, the known ways ${ }^{1-11}$ to derive free field equations are unsatisfactory in so far as they do not yield a background which motivates the introduction of field equations. The knowledge would be useful, for example, in general relativity if field equations in analogy to the known field equations in Minkowski space are to be derived.

We present a method for the derivation of field equations ${ }^{12}$ which permits calculating them in a natural way. As starting point we use that the motivation for the transformation law of fields in field theory has been claryfied nowadays in connection with the theory of G-bundels ${ }^{13-15}$, where induced representations ${ }^{16}$ of groups appear as a consequence of a profound geometrical concept. The field representations emerge in this context as representations of the Poincaré group with the Lorentz group as inducing subgroup.

The unitary and irreducible representations of the Poincaré group which have been classified by Wigner ${ }^{17}$, are induced representations too; however, the induction process is a different one. This suggests comparing these representations with the field representations. Such investigations - on the understanding that the field equations exist already have been done ${ }^{18}$.

We go the other way round - that is, forget the field equations - and interpret the field representation as autonomous. Which properties have these representations? As can easily be shown, they are neither unitary nor irreducible. Because these properties are "elementary" attributes of field theory,

Reprint requests to Dr. G. Grensing, Institut für Reine und Angewandte Kernphysik, D-2300 Kiel, Ohlshausenstrasse $40 / 60$. we investigate the question whether these representations may be restricted to a subspace on which they act unitarily and irreducibly.

This is indeed possible. We shall show how to calculate the restricting conditions for the representation space ${ }^{19}$. These are just the well known field equations. In this framework, the field equations are the necessary conditions to make the field representation unitary and irreducible.

Because the method is not restricted in its range of application it may be applied to more sophisticated problems, for example to the de Sitter model of general relativity ${ }^{20}$.

The field equations turn out to be not only necessary but even sufficient conditions for the field representation to be unitary and irreducible. We shall prove this by establishing the equivalence with the representation in Wignerian form. This offers a simple theory for solving the field equations, which allows to give the solutions for definite orientation of spin explicitely. For photons so far only properties of the solutions were known but not the solutions themselves. A further peculiarity of our procedure is that we generate the solutions with the help of a Wigner boost which contains only pure Lorentz transformations for finite as well as for vanishing mass. In the former case this is quite familiar, but in the latter it seems not to be known in the literature. This choice is advantageous because both cases - just as in the helicity formalism ${ }^{21}$ can be treated in a unique way.

For the massless vector field the statement that the field equations are even sufficient to guarantee unitarity and irreducibility is true only with some modification. In this case not the fields themselves carry a unitary and irreducible representation, but the equivalence classes of fields modulo gauge transformations. 
The field representation is a representation of the full Poincaré group, more exactly a representation of one of the eight covering groups of the full Poincaré group ${ }^{18,22}$. Hence, the field representation restricted by the field equations can act irreducibly only with respect to one of the covering groups of the full Poincaré group. It should be observed that the representation must be unitary; by no means the transformations containing the time inversion can be chosen to be antiunitary. In this case the restriction to the identity component would contain only positive energies. This is contradictious to the fact that the Klein-Gordon equation does contain also solutions with negative energy.

At first sight one might be inclined to think that the Wignerian theory - that is, the pure mathematical theory of unitary and irreducible representations of the Poincaré group - could be able to absorb free field theory completely. This impression is not correct, because the field representations restricted by the field equations contain more than the mere fact that they reproduce unitary and irreducible representations of the Poincaré group. For example they tell us that for particles with spin one half the discrete elements have to anticommute.

The paper is organized as follows: The second part collects the necessary basics, the only unusual features being the choice of the Wigner boost for mass zero and the effect of space inversion in a representation of one of the coverings of the full Poincaré group. The derivation of the field equations for particles with spin less than or equal to one is given in the third, the proof of unitarity and irreducibility of the field representations restricted by the field equations in the fourth part.

\section{Notation}

\section{The Lorentz and Poincaré Group}

The metric tensor $g$ over Minkowski space $M$ with elements $x=\left(x^{\mu}\right)_{\mu=0,1,2,3}$ is taken to be $g=\operatorname{diag}(+---)$. An element $\Lambda=\left({\Lambda^{\mu}}^{\mu}\right)_{\mu=0,1,2,3}$ of the Lorentz group $L$ maps $x$ into $x^{\prime}=\Lambda x$ such that the indefinite scalar product over $M$ is left invariant. The infinitesimal operators

$$
\left(M^{\mu \nu}\right) \varrho_{\tau}=i\left\{g^{\mu \varrho} g^{\nu}{ }_{\tau}-g^{u}{ }_{\tau} g^{\nu} \varrho\right\}
$$

of $L$ satisfy the commutation relations

$$
\begin{aligned}
{\left[M_{\mu \nu}, M_{Q \tau}\right]=} & -i\left\{g_{\mu Q} M_{\nu \tau}\right. \\
& \left.+g_{v \tau} M_{\mu Q}-g_{\mu \tau} M_{v Q}-g_{\nu Q} M_{\mu \tau}\right\} .
\end{aligned}
$$

As representatives of the decomposition of $L$ with respect to the identity component $L_{1}$ we use the identity, the space inversion, the time inversion and the total inversion:

$\Lambda_{1}=1, \quad \Lambda_{\mathrm{S}}=g, \quad \Lambda_{\mathrm{T}}=-g, \quad \Lambda_{\mathrm{ST}}=-\mathbf{1}$,

so that $\quad L=\sum_{\mathrm{J}} L_{1} \Lambda_{\mathrm{J}}, \quad \mathrm{J}={ }_{1, \mathrm{~S}, \mathrm{~T}, \mathrm{ST}}$.

An element of the Poincare group $P$ maps $x$ into $x^{\prime}=\Lambda x+$ a. In addition to the elements of the Lie algebra of $L$ the Lie algebra of $P$ contains the operators $P_{\mu}$ with commutation relations

$\left[M_{\mu \nu} P_{\tau}\right]=-i\left\{g_{\mu \tau} P_{\nu}-g_{v \tau} P_{\mu}\right\},\left[P_{\mu}, P_{v}\right]=0$.

The two Casimir operators of $P$ are

$$
C_{\mathrm{II}}=P_{\mu} P^{\mu}, \quad C_{\mathrm{IV}}=W_{\mu} W^{\mu}
$$

where $W^{\mu}=\frac{1}{2}, \varepsilon^{\mu \nu \varrho \tau} M_{v_{\rho}} P_{\tau}$. $W^{2}$ may be written in the form:

$$
\begin{aligned}
& W^{2}=-\left\{\frac{1}{2} M^{\mu \nu} M_{\mu \nu} P^{\tau} P_{\tau}+M^{\mu v} M_{\nu \tau} P_{\mu} P^{\tau}\right\} . \\
& \text { II. The Universal Covering Group of } L_{1} \text { and } P_{1}
\end{aligned}
$$

The universal covering group $\tilde{L_{1}}$ of $L_{1}$ acts as transformation group on $X=x^{\mu} \gamma_{\mu}$, where the $\gamma$-matrices obey the relation

$$
\gamma_{\mu} \gamma_{\nu}+\gamma_{\nu} \gamma_{\mu}=2 g_{\mu \nu} .
$$

They have the form

$$
\gamma^{\mu}=-\left(\begin{array}{ll}
0 & \tilde{\sigma}^{\mu} \\
\sigma^{\mu} & 0
\end{array}\right), \quad \begin{aligned}
& \tilde{\sigma}=\left(\mathbf{1},-\sigma^{k}\right)_{k=1,2,3} \\
& \sigma=\left(\mathbf{1},+\sigma^{k}\right)_{k=1,2,3}
\end{aligned}
$$

with $\sigma^{k}$ being the Pauli matrices.

An element $A$ of $\tilde{L}_{1}$ transforms $X$ into

$$
X^{\prime}=A X A^{-1}
$$

where $\quad A=\left(\begin{array}{ll}\mathcal{A} & 0 \\ 0 & \mathcal{A}^{\dagger-1}\end{array}\right)$ and $\mathcal{A} \in S L(2, C)$.

This defines the universal covering map

$$
\tilde{\pi}: S L(2, C) \rightarrow L_{1}
$$

which is explicitely given by

$$
\Lambda^{\mu}{ }_{\nu}=\frac{1}{2} \operatorname{Tr}\left\{\sigma^{\mu} \mathcal{A} \tilde{\sigma}_{v} \mathcal{A}^{\dagger}\right\} .
$$

Because $L_{1}$ is the part in $P_{1}$ which is not simply connected, the universal covering group $\tilde{P}_{1}$ of $P_{1}$ is known too.

\section{The Covering Groups of $L$ and $P$}

The Lorentz group is not connected, hence, several covering groups $\tilde{L}$ of $L$ exist $^{18,22}$. The analogue 
to $(2.3)$ is valid:

$$
\tilde{L}=\sum_{\mathrm{J}} \tilde{L}_{1} \mathcal{A}_{\mathrm{J}}, \quad \tilde{\pi}\left(A_{\mathrm{J}}\right)=\Lambda_{\mathrm{J}} .
$$

The multiplication for the elements of $\tilde{L}$ is determined by

$$
\begin{gathered}
\mathcal{A}_{\mathrm{J}} \mathcal{A}=\varrho_{\mathrm{J}}(\mathcal{A}) \mathcal{A}_{\mathrm{J}}, \\
\varrho_{\mathrm{S}}(\mathcal{A})=\mathcal{A}^{\dagger-1}=\varrho_{\mathrm{T}}(\mathcal{A}), \varrho_{1}(\mathcal{A})=\mathcal{A}=\varrho_{\mathrm{ST}}(\mathcal{A})
\end{gathered}
$$

and

\begin{tabular}{l|lllll} 
& $\mathbf{1}$ & $\mathcal{A}_{\mathrm{S}}$ & $\mathcal{A}_{\mathrm{T}}$ & $\mathcal{A}_{\mathrm{ST}}$ & \\
\hline $\mathbf{1}$ & $\mathbf{1}$ & $\mathcal{A}_{\mathrm{S}}$ & $\mathcal{A}_{\mathrm{T}}$ & $\mathcal{A}_{\mathrm{ST}}$ & \\
$\mathcal{A}_{\mathrm{S}}$ & $\mathcal{A}_{\mathrm{S}}$ & $\varepsilon_{\mathrm{S}} \mathbf{1}$ & $\mathcal{A}_{\mathrm{ST}}$ & $\varepsilon_{\mathrm{S}} \mathcal{A}_{\mathrm{T}}$ & $\varepsilon_{\mathrm{J}}= \pm 1$ \\
$\mathcal{A}_{\mathrm{T}}$ & $\mathcal{A}_{\mathrm{T}}$ & $\varepsilon_{\mathrm{S}} \varepsilon_{\mathrm{T}} \varepsilon_{\mathrm{ST}} \mathcal{A}_{\mathrm{ST}}$ & $\varepsilon_{\mathrm{T}} \mathbf{1}$ & $\varepsilon_{\mathrm{S}} \varepsilon_{\mathrm{ST}} \mathcal{A}_{\mathrm{S}}$ & \\
$\mathcal{A}_{\mathrm{ST}}$ & $\mathcal{A}_{\mathrm{ST}}$ & $\varepsilon_{\mathrm{T}} \varepsilon_{\mathrm{ST}} \mathcal{A}_{\mathrm{T}}$ & $\varepsilon_{\mathrm{T}} \mathcal{A}_{\mathrm{S}}$ & $\varepsilon_{\mathrm{ST}} \mathbf{1}$ & $(2.14)$
\end{tabular}

From (2.14) can easily be derived:

$$
\begin{gathered}
\mathcal{A}_{\mathrm{J}} \mathcal{A}_{\mathrm{J}^{\prime}}=\varepsilon \mathcal{A}_{\mathrm{J}^{\prime}} \mathcal{A}_{\mathrm{J}}, \varepsilon=\varepsilon_{\mathrm{S}} \varepsilon_{\mathrm{T}} \varepsilon_{\mathrm{ST}} \\
\mathrm{J} \neq \mathrm{J}^{\prime}, \mathrm{J}, \mathrm{J}^{\prime}=\mathrm{S}, \mathrm{T}, \mathrm{ST} .
\end{gathered}
$$

Thus, there exist eight non-isomorphic covering groups of $L$, four with commuting and four with anticommuting discrete elements. The problem to find the covering groups of $P$ has already been solved if those for $L$ are known.

$$
\text { IV. Unitary and Irreducible Representations of } \tilde{P}_{1}
$$

The complete system of unitary, irreducible representations of $\tilde{P}_{1}$ has been determined by Wigner ${ }^{17}$. We are only interested in the following series. $m>0$ )

The representations have the form

$$
\begin{gathered}
U(\mathrm{a}, \mathcal{A}) \varphi\left(p_{ \pm}\right)=e^{i \mathrm{a} p_{ \pm}} D^{(s)}\left(\mathcal{A}_{p_{ \pm}}^{-1} \mathcal{A} \mathcal{A}_{\Lambda^{-1} p_{ \pm}}\right) \varphi\left(\Lambda^{-1} p_{ \pm}\right) \\
p_{ \pm} \in H_{m>0}^{ \pm}=\left\{p \in M \mid p^{2}=m^{2}>0, p^{0} \gtrless 0\right\} \\
\Lambda=\tilde{\pi}(\mathcal{A})
\end{gathered}
$$

where $\dot{\mathcal{A}}\left(\mathcal{A}, p_{ \pm}\right)=\mathcal{A}_{p_{ \pm}}^{-1} \mathcal{A} \mathcal{A}_{\Lambda^{-1} p_{ \pm}}$is an element of $S U(2)$, the stationary subgroup of $\dot{p}_{ \pm}=( \pm m, 0,0,0)$, and $D^{(s)}$ a unitary, irreducible representation of $S U(2)$ with $s=0, \frac{1}{2}, 1, \frac{3}{2}, \ldots$ A usual choice of the Wigner boost is

$$
\mathcal{A}_{p_{ \pm}}=\frac{1}{\sqrt{2 m(\omega+m)}}\left(\begin{array}{ll}
m+\omega \pm p^{3} & \pm\left(p^{1}-i p^{2}\right) \\
\pm\left(p^{1}+i p^{2}\right) & m+\omega \mp p^{3}
\end{array}\right), \quad \begin{aligned}
& \omega=\sqrt{m^{2}+\vec{p}^{2}} \\
& \mathcal{A}_{p_{ \pm}}^{\dagger}=\mathcal{A}_{p_{ \pm}}
\end{aligned}
$$

and the corresponding element in $L_{1}$

$$
\Lambda_{p_{ \pm}}=\left(\begin{array}{cl}
\frac{\omega}{m} & \mp \frac{p_{i}}{m} \\
\pm \frac{p^{k}}{m} & g_{i}^{k}-\frac{p^{k} p_{i}}{m(\omega+m)}
\end{array}\right)_{k, i=1,2,3} \Lambda_{p_{ \pm}}^{\dagger}=\Lambda_{p_{ \pm}} .
$$

It contains only pure Lorentz transformations. The representation $U$ is unitary with respect to the scalar product

$$
\left(\varphi, \varphi^{\prime}\right)=\int_{H_{m>0}^{ \pm}} \mathrm{d} \mu\left(p_{ \pm}\right) \sum_{s_{3}=-s}^{+s} \varphi^{*}\left(p_{ \pm}, s_{3}\right) \varphi^{\prime}\left(p_{ \pm}, s_{3}\right)
$$

where

$$
\varphi\left(p_{ \pm}\right)=\sum_{s_{3}} \varphi\left(p_{ \pm}, s_{3}\right) e_{s_{3}}^{ \pm} \quad \text { and } \quad \mathrm{d} \mu\left(p_{ \pm}\right)=\frac{\mathrm{d}^{3} p}{\omega}=\mathrm{d} \mu\left(\Lambda p_{ \pm}\right)
$$

is the invariant measure over $H \frac{ \pm}{m}$. The eigenvalues of the Casimir operators are:

$$
U\left(C_{\mathrm{II}}\right)=m^{2}, \quad U\left(C_{\mathrm{IV}}\right)=-m^{2} s(s+1) .
$$

These representations we label by $[ \pm m, s]$.

$m=0$ )

In this case

where

$$
U(\mathrm{a}, \mathcal{A}) \varphi\left(p_{ \pm}\right)=e^{i \mathrm{a} p_{ \pm}} \exp \left\{i s \alpha\left(\mathcal{A}, p_{ \pm}\right)\right\} \varphi\left(\Lambda^{-1} p_{ \pm}\right)
$$

and $\dot{\mathcal{A}}\left(\mathcal{A}, p_{ \pm}\right)=\mathcal{A}_{p_{ \pm}}^{-1} \mathcal{A} \mathcal{A}_{\Lambda^{-1} p_{ \pm}}$is an element of $E(2)$, the stationary subgroup of $\dot{p}_{ \pm}=( \pm 1,0,0,1)$ :

$$
\begin{array}{ccc}
\dot{\mathcal{A}}_{+}=\left(\begin{array}{ll}
\exp \left\{+i \alpha_{+} / 2\right\} & \exp \left\{-i \alpha_{+} / 2\right\} w_{+} \\
0 & \exp \left\{-i \alpha_{+} / 2\right\}
\end{array}\right) & \dot{\mathcal{A}}_{-}=\left(\begin{array}{ll}
\exp \left\{-i \alpha_{-} / 2\right\} & 0 \\
\exp \left\{-i \alpha_{-} / 2\right\} w_{-} & \exp \left\{+i \alpha_{-} / 2\right\}
\end{array}\right) \\
w_{ \pm}=u_{ \pm}+i v_{ \pm}, 0 \leqq \alpha_{ \pm} \leqq 4 \pi .
\end{array}
$$


With the help of (2.11) :

$$
\dot{\Lambda}_{ \pm}=\left(\begin{array}{cccc}
1+\frac{\left|w_{ \pm}\right|^{2}}{2}+\left(\cos \alpha_{ \pm} u_{ \pm}+\sin \alpha_{ \pm} v_{ \pm}\right) & \mp\left(-\sin \alpha_{ \pm} u_{ \pm}\right. & \left.+\cos \alpha_{ \pm} v_{ \pm}\right) \mp \frac{\left|w_{ \pm}\right|^{2}}{2} \\
u_{ \pm} & \cos \alpha_{ \pm} & \pm \sin \alpha_{ \pm} & \mp u_{ \pm} \\
\mp v_{ \pm} & \mp \sin \alpha_{ \pm} & \cos \alpha_{ \pm} & v_{ \pm} \\
\pm \frac{\left|w_{ \pm}\right|^{2}}{2} & \pm\left(\cos \alpha_{ \pm} u_{ \pm}+\sin \alpha_{ \pm} v_{ \pm}\right) & -\left(-\sin \alpha_{ \pm} v_{ \pm}+\cos \alpha_{ \pm} v_{ \pm}\right) 1-\frac{\left|w_{ \pm}\right|^{2}}{2}
\end{array}\right) .
$$

$D^{(s)}\left(\dot{\mathcal{A}}_{ \pm}\right)=e^{i s \alpha_{ \pm}}$is a one-dimensional, unitary and irreducible representation of the stationary subgroup with $s=0, \pm \frac{1}{2}, \pm 1, \pm \frac{3}{2}, \ldots$ As Wigner boost we choose a matrix which establishes the greatest possible similarity of the representations with $m>0$ and $m=0$ :

$$
\mathcal{A}_{p_{+}}=\frac{1}{\sqrt{2\left(\omega+p^{3}\right)}}\left(\begin{array}{ll}
\omega+p^{3} & p^{1}-i p^{2} \\
p^{1}+i p^{2} & 2+\omega-p^{3}
\end{array}\right) \quad \begin{gathered}
\mathcal{A}_{p-}=\mathcal{A}_{p_{+}}^{-1} \\
\mathcal{A}_{p_{ \pm}}^{\dagger}=\mathcal{A}_{p_{ \pm}}
\end{gathered} .
$$

Again it contains only pure Lorentz transformations. The computation of the corresponding Lorentz transformation with the help of (2.11) yields:

$$
\Lambda_{p_{ \pm}}=\frac{1}{\omega+p^{3}}\left(\begin{array}{rlrr}
-\left(p^{3}-1\right)+(\omega+1) \omega & \mp(\omega+1) p_{1} & \mp(\omega+1) p_{2} \pm\left(p^{3}-1\right) \pm\left(p^{3}-1\right) \omega \\
\pm(\omega+1) p^{1} & \left(\omega+p^{3}\right)-p^{1} p_{1} & -p^{1} p_{2} & \left(p^{3}-1\right) p^{1} \\
\pm(\omega+1) p^{2} & -p^{2} p_{1} & \left(\omega+p^{3}\right)-p^{2} p_{2} & \left(p^{3}-1\right) p^{2} \\
\mp(\omega+1) \pm(\omega+1) p^{3} & -\left(p^{3}-1\right) p_{1} & -\left(p^{3}-1\right) p_{2} & (\omega+1)+\left(p^{3}-1\right) p^{3}
\end{array}\right) .
$$

The scalar product has the same form with $s_{3}=s$ as has been given in (2.19). The Casimir operators both have the eigenvalue zero. These representations we label by $[ \pm 0, s]$.

\section{$V$. Representations of $\tilde{L}$ and $\tilde{P}$}

The linear, irreducible and finite dimensional resp. unitary representations of $\tilde{L}$ resp. $\tilde{P}$ can be obtained with the help of a theorem due to Clifford ${ }^{23}$.

The finite dimensional, irreducible representations of $\tilde{L}$ have been studied by Gelfand, Minlos and Shapiro ${ }^{8}$. Their results differ from ours. We only list the inequivalent representations of dimension less than or equal to four:

0) $D(\mathcal{A})=\mathbf{1}$

$D\left(\mathcal{A}_{\mathrm{S}}\right)=\hat{\varepsilon}_{\mathrm{S}} \mathbf{1}, \quad D\left(\mathcal{A}_{\mathrm{T}}\right)=\hat{\varepsilon}_{\mathrm{T}} \mathbf{1}, \quad \boldsymbol{D}\left(\mathcal{A}_{\mathrm{ST}}\right)=\hat{\varepsilon}_{\mathrm{S}} \hat{\varepsilon}_{\mathrm{T}} \mathbf{1}$

$\left(\varepsilon_{\mathrm{S}}, \varepsilon_{\mathrm{T}}, \varepsilon_{\mathrm{ST}}\right)$ is arbitrary and $\hat{\varepsilon}_{\mathrm{S}}, \hat{\varepsilon}_{\mathrm{T}}= \pm 1$.

1) $D(\mathcal{A})=A$

\begin{tabular}{l|cccc}
$\left(\varepsilon_{\mathrm{S}}, \varepsilon_{\mathrm{T}}, \varepsilon_{\mathrm{ST}}\right)$ & $A_{\mathrm{S}}$ & $A_{\mathrm{T}}$ & $A_{\mathrm{ST}}$ \\
\hline$(+,-,+)$ & $\gamma^{0}$ & $\hat{\varepsilon}_{\mathrm{T}} \gamma^{0} \gamma^{5}$ & $\hat{\varepsilon}_{\mathrm{T}} \gamma^{5}$ & $\hat{\varepsilon}_{\mathrm{T}}= \pm 1$ \\
$(+,+,-)$ & $\gamma^{0}$ & $i \hat{\varepsilon}_{\mathrm{T}} \gamma^{0} \gamma^{5}$ & $i \hat{\varepsilon}_{\mathrm{T}} \gamma^{5}$ & $\gamma^{5}=i \gamma^{0} \gamma^{1} \gamma^{2} \gamma^{3}$
\end{tabular}

The only property of the representations with commuting discrete elements we need is that $A_{\mathrm{ST}}$ is a multiple of the identity.
1) $D(\mathcal{A})=\pi(\mathcal{A})=\Lambda, \quad D\left(\mathcal{A}_{\mathrm{S}}\right)=\hat{\varepsilon}_{\mathrm{S}} \Lambda_{\mathrm{S}}$,

$$
D\left(A_{\mathrm{T}}\right)=\hat{\varepsilon}_{\mathrm{T}} \Lambda_{\mathrm{T}}, D\left(A_{\mathrm{ST}}\right)=\hat{\varepsilon}_{\mathrm{S}} \hat{\varepsilon}_{\mathrm{T}} \Lambda_{\mathrm{ST}}
$$

$\left(\varepsilon_{\mathrm{S}}, \varepsilon_{\mathrm{T}}, \varepsilon_{\mathrm{ST}}\right)$ is arbitrary and $\hat{\varepsilon_{\mathrm{S}}}, \hat{\varepsilon}_{\mathrm{T}}= \pm 1$.

The antiunitary ray representations of $P$ have been investigated extensively ${ }^{7}, 18,24$. We shall see, however, that in our framework it is only meaningful to deal with fields which transform under unitary representations of $\tilde{P}$. Investigations of this sort of representations are rare and detailed only for $m>0$. Therefore, we list here the system of those unitary and irreducible representations of $\tilde{P}$ which contain representations of $\tilde{P}_{1}$ with $p_{ \pm}^{2}=m^{2} \geqq 0$ if restricted to $\tilde{P}_{1}$ :

$m>0$ )

The restriction to $\tilde{P}_{1}$ decomposes into the direct sum with positive and negative energy:

$$
\begin{aligned}
& U(\mathrm{a}, \mathcal{A}) \sum_{s_{s}} \varphi\left(p_{ \pm}, s_{3}\right) e_{s_{3}}^{ \pm} \\
& =e^{i \mathrm{a} p_{ \pm}} \sum_{s_{S}{ }^{s_{s}}} D^{(s)}\left(\mathcal{A}_{p_{ \pm}}^{-1} \mathcal{A} \mathcal{A}_{\Lambda^{-1} p_{ \pm}}\right)_{s_{3} s_{3}^{\prime}} \varphi\left(\Lambda^{-1} p_{ \pm}, s_{3}{ }^{\prime}\right) e_{s_{3}}^{ \pm} \\
& \begin{aligned}
U\left(\mathcal{A}_{\mathrm{S}}\right) \sum_{s_{3}} \varphi\left(p_{+}, s_{3}\right) e_{s_{s}}^{+} \\
=\pi\left(\varepsilon_{\mathrm{S}}\right)^{s} \sum_{s_{3}} \varphi\left(\Lambda_{\mathrm{S}}{ }^{-1} p_{+}, s_{3}\right) e_{s_{3}}^{+}
\end{aligned}
\end{aligned}
$$




$$
\begin{aligned}
& U\left(\mathcal{A}_{\mathrm{S}}\right) \sum_{s_{3}} \varphi\left(p_{-}, s_{3}\right) e_{s_{3}}^{-} \\
& U\left(\mathcal{A}_{\mathrm{ST}}\right)=U\left(\mathcal{A}_{\mathrm{S}}\right) U\left(\mathcal{A}_{\mathrm{T}}\right) \quad \pi= \pm 1 . \\
& \left.=\pi(\varepsilon)^{2 s}\left(\varepsilon_{\mathrm{S}}\right)^{s} \sum_{s_{3}} \varphi\left(\Lambda_{\mathrm{S}}{ }^{-1} p_{-}, s_{3}\right) e_{s_{3}}^{-} \quad m=0\right) \\
& U\left(\mathcal{A}_{\mathrm{T}}\right) \sum_{s_{\mathrm{s}}} \varphi\left(p_{+}, s_{3}\right) \boldsymbol{e}_{s_{\mathrm{s}}}^{+}=\sum_{s_{\mathrm{s}}} \varphi\left(\Lambda_{\mathrm{T}}^{-1} \boldsymbol{p}_{+}, s_{3}\right) e_{s_{3}}^{-}
\end{aligned}
$$$$
U\left(\mathcal{A}_{\mathrm{T}}\right) \sum_{s_{3}} \varphi\left(p_{-}, s_{3}\right) \boldsymbol{e}_{s_{3}}^{-}
$$

The restriction to $\tilde{P}_{1}$ contains in addition to a representation with helicity $+s$ the representation with helicity $-s$ and together with a representation with positive energy the representation with nega$=\left(\varepsilon_{\mathrm{T}}\right)^{2 s} \sum_{s_{3}} \varphi\left(\Lambda_{\mathrm{T}}^{-1} p_{-}, s_{3}\right) e_{s_{3}}^{+}$ tive energy:

$$
\begin{aligned}
& U(\mathrm{a}, \mathcal{A})\left[\varphi\left(p_{ \pm}, s_{3}\right) e_{s_{\mathrm{s}}}^{ \pm}\right]=e^{i \mathrm{a} p_{ \pm}} \exp \left\{i s_{3} \alpha(\mathcal{A}, p \pm)\right\} \varphi\left(\Lambda^{-1} p_{ \pm}, s_{3}\right) e_{s_{3}}^{ \pm} \\
& s_{3}= \pm s \quad s=0, \frac{1}{2}, 1, \frac{3}{2}, \ldots \\
& U\left(\mathcal{A}_{\mathrm{S}}\right)\left[\varphi\left(p_{+},+s\right) e_{+s}^{+}\right]=e^{-i s_{\chi_{+}}} \varphi\left(\Lambda_{\mathrm{S}}^{-1} p_{+},+s\right) e_{-s}^{+} \\
& U\left(\mathcal{A}_{\mathrm{S}}\right)\left[\varphi\left(p_{+},-s\right) e_{-s}^{+}\right]=\left(-\varepsilon_{\mathrm{S}}\right)^{2 s} e^{+i s_{\chi_{+}}} \varphi\left(\Lambda_{\mathrm{S}}^{-1} p_{+},-s\right) e_{+s}^{+} \\
& U\left(\mathcal{A}_{\mathrm{S}}\right)\left[\varphi\left(p_{-},+s\right) e_{+s}^{-}\right]=(\varepsilon)^{2 s} e^{-i s_{-}} \varphi\left(\Lambda_{\mathrm{S}}^{-1} p_{-},+s\right) e_{-s}^{-} \\
& U\left(\mathcal{A}_{\mathrm{S}}\right)\left[\varphi\left(p_{-},-s\right) e_{-s}^{-}\right]=\left(-\varepsilon \varepsilon_{\mathrm{S}}\right)^{2 s} e^{+i s_{-}} \varphi\left(\Lambda_{\mathrm{S}}^{-1} p_{-},-s\right) e_{+s}^{-} \\
& e^{+i \chi_{+} / 2}=-p^{1}+i p^{2} / \sqrt{\left(\omega+p^{3}\right)\left(\omega-p^{3}\right)}=e^{-i \chi_{-} / 2} \\
& U\left(\mathcal{A}_{\mathrm{T}}\right)\left[\varphi\left(p_{+}, \pm s\right) e_{ \pm s}^{+}\right]=\varphi\left(\Lambda_{\mathrm{T}}^{-1} p_{+}, \pm s\right) e_{\mp s}^{-} \\
& U\left(\mathcal{A}_{\mathrm{T}}\right)\left[\varphi\left(p_{-}, \pm s\right) e_{ \pm s}^{-}\right]=\left(\varepsilon_{\mathrm{T}}\right)^{2 s} \varphi\left(\Lambda_{\mathrm{T}}^{-1} p_{-}, \pm s\right) e_{\mp s}^{+} \\
& U\left(\mathcal{A}_{\mathrm{ST}}\right)=U\left(\mathcal{A}_{\mathrm{S}}\right) U\left(\mathcal{A}_{\mathrm{T}}\right) .
\end{aligned}
$$

These formulas remain unchanged in the helicity formalism.

\section{Field Representations}

\section{Induced Representations and Fields}

Let $G$ be a transitive Lie transformation group on the manifold $M$ and $\dot{G}$ the stationary subgroup of the point $\dot{x}$ of $M$. Because $M$ and $G / \dot{G}$ are diffeomorphic, the decomposition of $G$ with respect to $\dot{G}$ may be written:

$$
G=\sum_{x \in M} g_{x} \dot{G}, \quad g_{x} \dot{x}=x .
$$

The representation $T$ of $G$, which is induced by the unitary representation $D$ of $\dot{G}$ on $V$, acts on $L^{2}(M, \mu, V)$ and is defined as follows ${ }^{25}$ :

$T(g) \psi(x)=\left\{\frac{\mathrm{d} \mu\left(g^{-1} x\right)}{\mathrm{d} \mu(x)}\right\}^{\frac{1}{2}} D\left(g_{x}^{-1} g g_{g^{-1} x}\right) \psi\left(g^{-1} x\right)$.

It is unitary with respect to

$$
(\psi, \chi)=\int_{M} \mathrm{~d} \mu(x)\langle\psi(x), \chi(x)\rangle
$$

where $\langle$,$\rangle is the scalar product over V$ and $\mu$ the measure over $M$.

The representation (3.2) can be shown to be equivalent to the version of induced representations given by Mackey ${ }^{16}$. It is well known that the series of unitary and irreducible representations of $\tilde{P}_{1}$ are obtained just in this way [cf. (2.16) and (2.21)].

We change the concept in one point and do not require $D$ to be unitary. Then of course $T$ is no longer unitary.

If we choose as manifold the Minkowski space $M$ and as transformation group the Poincare group $\tilde{P}$ we get well known representations: The stationary subgroup of $\dot{x}=0$ is the Lorentz group $\tilde{L}$, hence $\tilde{P} / \tilde{L}$ is diffeomorphic to $M$. If $D$ is a finite dimensional, irreducible representation of $\tilde{L}$ we get

$$
\begin{aligned}
T(\mathrm{a}, \mathcal{A}) \psi(x) & =D(\mathcal{A}) \psi\left(\Lambda^{-1}(x-\mathrm{a})\right) \\
T\left(\mathcal{A}_{\mathrm{J}}\right) \psi(x) & =D\left(\mathcal{A}_{\mathrm{J}}\right) \psi\left(\Lambda_{\mathrm{J}}^{-1} x\right) .
\end{aligned}
$$

This is the transformation law of fields as it ap. pears in relativistic field theory. Because (3.2) follows from the geometrical concept of $G$-bundles ${ }^{13-15}$, we are of opinion that this is the framework which is at the bottom of the transformation law of fields.

Of course, the field representation (3.3) of $\tilde{P}$ is neither unitary nor irreducible. We examine the question whether the representation space can be restricted to a subspace on which the representation acts unitarily and irreducibly. We shall demonstrate that this can be done indeed. The restricting con- 
ditions for the function space have just the form of field equations.

The field equations thus enforce the field representation to become a unitary and irreducible representation of the Poincaré group.

\section{Field Equations}

If the field representation is to be restricted to a unitary and irreducible representation, evidently first the condition has to be satisfied that the Casimir operators act as a multiple of the identity operator. Using the infinitesimal operators

$$
\begin{aligned}
T\left(P_{\mu}\right) & =i \partial_{\mu}, \\
T\left(M_{\mu \nu}\right) & =D\left(M_{\mu \nu}\right)+i\left(x_{\mu} \partial_{\nu}-x_{\nu} \partial_{\mu}\right)
\end{aligned}
$$

the invariants (2.5) take the form

$$
\begin{aligned}
T\left(C_{\mathrm{II}}\right) & =-\partial_{\mu} \partial^{\mu}, \\
T\left(C_{\mathrm{IV}}\right) & =\frac{1}{2} D\left(M^{\mu \nu}\right) D\left(M_{\mu \nu}\right) \partial_{\tau} \partial_{\tau} \\
& +D\left(M^{\mu \nu}\right) D\left(M_{\nu \tau}\right) \partial_{\mu} \partial^{\tau} .
\end{aligned}
$$

Hence we have to require:

a)

$$
T\left(C_{\mathrm{II}}\right)=m^{2} .
$$

This is the Klein-Gordon equation

$$
\left(\partial_{\mu} \partial^{\mu}+m^{2}\right) \psi(x)=0 .
$$

b)

$$
T\left(C_{\mathrm{IV}}\right)=-m^{2} s(s+1) .
$$

We only examine the representations $(2.26),(2.27)$ and (2.28). The case of arbitrary spin will be dealt with in a later publication.

0) $D(\mathcal{A})=\mathbf{1}$

We get $T\left(C_{\mathrm{IV}}\right)=0$, so that $s=0$ is necessary for $m>0$.

表) $D(A)=A$

With the infinitesimal operators $D\left(M_{\mu \nu}\right)=$ $i / 2\left(\gamma_{\mu} \gamma_{v}-g_{\mu \nu}\right)$ we get $T\left(C_{\mathrm{IV}}\right)=-\frac{3}{4} m^{2}$, which requires $s=\frac{1}{2}$ for $m>0$.

1) $D(A)=A$

By means of (2.1) one can easily compute:

$$
\left[T\left(C_{\mathrm{IV}}\right) \psi\right]^{\mu}(x)=-2 m^{2} \psi^{\mu}(x)-2 \partial^{\mu} \partial_{\nu} \psi^{\nu}(x) .
$$

The second invariant is no multiple of the identity in this case. We can achieve it however if $\partial^{\mu} \partial_{\nu} \psi^{\nu}(x)=0$ is required, this yielding the Lorentz convention for $s=1$ and $m>0$ :

$$
\partial_{\mu} \psi^{\mu}(x)=0 \text {. }
$$

For $m=0$ it can be shown that (3.9) is necessary too.
We shall prove that for $s=0$ the Klein-Gordon equation and for $s=1$ the Klein-Gordon equation together with the Lorentz convention suffice to guarantee the unitarity and irreducibility of the field representations.

The field representation for $s=\frac{1}{2}$ with the KleinGordon equation as subsidiary condition, however, is not yet irreducible. In this case we try to find additional commuting operators. If such an operator exists Schur's lemma suggests the following procedure: We try to enforce unitarity and irreducibility by means of the requirement that this operator acts as a multiple of the identity.

Because the infinitesimal operators act as differential operators, it suggests looking for invariant differential operators. We try to do the end with the simplest type, the differential operator of first order

$$
C^{\mu}(x) \partial_{\mu}+C(x)
$$

where $C^{\mu}(x)$ and $C(x)$ are elements of $L(V, V)$.

The invariance condition reads

$$
\left[C^{\mu}(x) \partial_{\mu}+C(x), T\left(\mathrm{a}, \mathcal{A} \mid \mathcal{A}_{\mathrm{J}}\right)\right]=0 .
$$

The commutativity with the translations requires the matrices to be constant over $M$ and the commutativity with the homogeneous transformations:

$$
\begin{aligned}
{\left[C, D\left(\mathcal{A} \mid \mathcal{A}_{\mathrm{J}}\right)\right] } & =0, \\
D(\mathcal{A}) C^{\mu} D^{-1}(\mathcal{A}) & ={A_{v}}^{\mu} C^{v}, \\
D\left(\mathcal{A}_{\mathrm{J}}\right) C^{\mu} D^{-1}\left(\mathcal{A}_{\mathrm{J}}\right) & =\Lambda_{\mathrm{J} v}{ }^{\mu} C^{v} .
\end{aligned}
$$

Because the representation $D$ of $\tilde{L}$ is irreducible, we can assume that the invariant operator is a pure differential operator with $C=0$. The remaining conditions require the $\left(C^{\mu}\right)_{\mu=0,1,2,3}$ to transform as a four-vector under the representation $D$ of the complete Lorentz group $\tilde{L}$.

The application to $s=\frac{1}{2}$ is simple: (2.9) shows that the $\gamma$-matrices behave as a four-vector with respect to $\tilde{L}_{1}$. Because $\gamma^{5}$ commutes with $A$, $\left(\gamma^{5} \gamma^{\mu}\right)_{\mu=0,1,2,3}$ is a four-vector too. It can be proved that these are indeed the only ones.

Furthermore it is readily verified with the help of (2.27) that $\left(C^{\mu}=\gamma^{\mu}\right)_{\mu=0,1,2,3}$ is a four-vector even for the anticommuting discrete transformations. This holds not true for $\left(C^{\mu}=\gamma^{5} \gamma^{\mu}\right)_{\mu=0,1,2,3}$, a four-vector for commuting discrete elements does not exist. Thus we require:

$$
\gamma^{\mu} \partial_{\mu} \psi(x)=\lambda \psi(x) .
$$

The constant $\lambda$ takes the values $\pm i m$, as can easily be seen by twofold application of the invariant 
operator. The sign is irrelevant, we choose:

$$
\left(i \gamma^{\mu} \partial_{\mu}-m\right) \psi(x)=0 \text {. }
$$

This is just the Dirac equation.

\section{Proof of Unitarity and Irreducibility}

\section{Equivalence of Field Representations and Wignerian Representations}

To establish the equivalence to unitary irreducible representations we recall that in each case the KleinGordon equation has to be satisfied, the solutions of which we write

$$
\begin{aligned}
\psi(x)=\int \mathrm{d} \mu\left(p_{+}\right) e^{-i p_{+} x} \psi\left(p_{+}\right) \\
+\int \mathrm{d} \mu\left(p_{-}\right) e^{-i p_{-} x} \psi\left(p_{-}\right)
\end{aligned}
$$

with arbitrary $\psi\left(p_{+}\right)$and $\psi\left(p_{-}\right)$. They inherit the transformation law

$$
\begin{aligned}
T(\mathrm{a}, \mathcal{A}) \psi\left(p_{ \pm}\right) & =e^{i \mathrm{a} p_{ \pm}} D(\mathcal{A}) \psi\left(\Lambda^{-1} p_{ \pm}\right), \\
T\left(\mathcal{A}_{\mathrm{J}}\right) \psi\left(p_{ \pm}\right) & =D\left(\mathcal{A}_{\mathrm{J}}\right) \psi\left(\Lambda_{\mathrm{J}}^{-1} p_{ \pm}\right) .
\end{aligned}
$$

The restriction of this representation to $\tilde{P}_{1}$ is equi- valent to a representation in Wignerian form ${ }^{7}$ : To prove this, we define the function $\varphi\left(p_{ \pm}\right)$by

$$
\psi\left(p_{ \pm}\right)=D\left(\mathcal{A}_{p_{ \pm}}\right) \varphi\left(p_{ \pm}\right) .
$$

With the help of (4.2 a) we obtain

$$
\begin{aligned}
\varphi\left(p_{ \pm}\right) \rightarrow & U(\mathrm{a}, \mathcal{A}) \varphi\left(p_{ \pm}\right) \\
& =e^{i \mathrm{a} p_{ \pm}} D\left(\mathcal{A}_{p_{ \pm}}^{-1} \mathcal{A} \mathcal{A}_{\Lambda^{-1} p_{ \pm}}\right) \varphi\left(\Lambda^{-1} p_{ \pm}\right)
\end{aligned}
$$

which is the required result.

Thus we may deduce: Suppose, a field representation is induced by a representation of $S L(2, \mathrm{C})$ which is irreducible with respect to $S U(2)$. If the Klein-Gordon equation with $m>0$ is imposed as subsidiary condition and in addition negative energies are excluded this representation is equivalent to a unitary, irreducible representation of $\tilde{P}_{1}$. Consequently, a nontrivial situation can only arise if one aims at field representations of the full Poincaré group.

As we shall show, the remaining field equations restrict $\psi\left(p_{ \pm}\right)$for $\dot{p}_{ \pm}=p_{ \pm}$to solutions $e\left(\dot{p}_{ \pm}, s_{3}\right)$ which transform under an element of the stationary subgroup as follows:

$$
\begin{array}{lll}
m>0: & D\left(\dot{\mathcal{A}}_{ \pm}\right) e\left(\dot{p}_{ \pm}, s_{3}\right)=\sum_{s_{3}{ }^{\prime}} D^{(s)}\left(\dot{\mathcal{A}}_{ \pm}\right)_{s_{3}{ }^{\prime} s_{3}} e\left(\dot{p}_{ \pm}, s_{3}{ }^{\prime}\right) & s_{3}=-s, \ldots,+s, \\
m=0: & D\left(\dot{\mathcal{A}}_{ \pm}\right) e\left(\dot{p}_{ \pm}, s_{3}\right)=e^{i s_{3} \alpha_{ \pm}} e\left(\dot{p}_{ \pm}, s_{3}\right) & s_{3}=-s,+s .
\end{array}
$$

For $m=0$ this is only valid with some unessential modification which will be given below.

Hence we obtain the result that the representation $(4.2 \mathrm{a})$ is equivalent to $[ \pm m, s]$ for $m>0$ resp. to $[ \pm 0,+s] \oplus[ \pm 0,-s]$ for $m=0$ where $s=$ $0, \frac{1}{2}, 1$.

With the help of (4.3) we are able to write the general $\psi\left(\boldsymbol{p}_{ \pm}\right)$in the form

$$
\psi\left(p_{ \pm}\right)=\sum_{s_{3}} \varphi\left(p_{ \pm}, s_{3}\right) e\left(p_{ \pm}, s_{3}\right),
$$

where we have defined

$$
e\left(p_{ \pm}, s_{3}\right)=D\left(\mathcal{A}_{p_{ \pm}}\right) e\left(\dot{p}_{ \pm}, s_{3}\right) .
$$

This reduces the problem to solve the field equations to a fairly easy problem. We shall see that (4.8) yields exactly the same solution in those cases which are already known.

The representation $T$ inherits from $U$ the unitary scalar product

$$
\begin{aligned}
\left(\psi, \psi^{\prime}\right)=\int \mathrm{d} \mu\left(p_{+}\right)\left\langle\psi\left(p_{+}\right), \psi^{\prime}\left(p_{+}\right)\right\rangle \\
+\int \mathrm{d} \mu\left(p_{-}\right)\left\langle\psi\left(p_{-}\right), \psi^{\prime}\left(p_{-}\right)\right\rangle
\end{aligned}
$$

where

$\left\langle\psi\left(p_{ \pm}\right), \psi^{\prime}\left(p_{ \pm}\right)\right\rangle=\psi^{\dagger}\left(p_{ \pm}\right) D^{\dagger}\left(\mathcal{A}_{p_{ \pm}}^{-1}\right) D\left(\mathcal{A}_{p_{ \pm}}^{-1}\right) \psi^{\prime}\left(p_{ \pm}\right)$.

About the discrete transformations nothing can be stated with such a generality: We anticipate that indeed they make the field representation a unitary and irreducible representation of $\tilde{P}$. Both for $m>0$ and for $m=0$ the arbitrariness in the choice of $\hat{\varepsilon}_{\mathrm{T}}$ leads to equivalent representations of $\tilde{P}$. If $m=0$ and $s \neq 0$ this is equally valid for those representations of $\tilde{L}$ which differ in the choice of $\hat{\varepsilon}_{S}$. For the sake of brevity we confine ourselves to the following representations of the discrete transformations:

\begin{tabular}{l|lll} 
& $\left(\varepsilon_{\mathrm{S}}, \varepsilon_{\mathrm{T}}, \varepsilon_{\mathrm{ST}}\right)$ & $D\left(\mathcal{A}_{\mathrm{S}}\right)$ & $D\left(\mathcal{A}_{\mathrm{T}}\right)$ \\
\hline$s=0$ & arbitrary & $\mathbf{1}$ & $\mathbf{1}$ \\
$s=\frac{1}{2}$ & $(+,-,+)$ & $\gamma^{0}$ & $\gamma^{0} \gamma^{\mathbf{5}}$ \\
$s=1$ & arbitrary & $\Lambda_{\mathrm{S}}$ & $\Lambda_{\mathrm{T}}$
\end{tabular}


$s=0$ :

\section{I. Proof of Equivalence}

This representation is appearently unitary and irreducible as a representation of $\tilde{P}$; the unitary scalar product is :

$\left(\psi, \psi^{\prime}\right)=\int \mathrm{d} \mu\left(p_{+}\right) \psi^{*}\left(p_{+}\right) \psi^{\prime}\left(p_{+}\right)$

$s=\frac{1}{2}:$

$$
+\int \mathrm{d} \mu\left(p_{-}\right) \psi^{*}\left(p_{-}\right) \psi^{\prime}\left(p_{-}\right) \text {. }
$$

From the representation (2.8) we change to an equivalent one by means of

$$
\begin{aligned}
& e\left(\dot{p}_{+},+\frac{1}{2}\right)=\left(\begin{array}{l}
1 \\
0 \\
0 \\
0
\end{array}\right), \quad e\left(\dot{p}_{+},-\frac{1}{2}\right)=\left(\begin{array}{l}
0 \\
1 \\
0 \\
0
\end{array}\right) \\
& \dot{\mathcal{A}}) e\left(\dot{p}_{ \pm}, s_{3}\right)=\sum_{s_{3}^{\prime}} D^{\left(\frac{1}{2}\right)}(\dot{\mathcal{A}})_{s_{3}{ }^{\prime} s_{3}} e\left(\dot{p}_{ \pm}, s_{3}{ }^{\prime}\right)
\end{aligned}
$$

for $\dot{A}$ in $S U(2)$, hence

$U(\mathrm{a}, \mathcal{A}) \varphi\left(p_{ \pm}\right)$

$$
\begin{gathered}
=e^{i \mathrm{a} p_{ \pm}} D^{\left(\frac{1}{2}\right)}\left(\mathcal{A}_{p_{ \pm}}^{-1} \mathcal{A} \mathcal{A}_{\Lambda^{-1} p_{ \pm}}\right) \varphi\left(\Lambda^{-1} p_{ \pm}\right) \\
\varphi\left(p_{ \pm}\right)=\sum_{s_{3}= \pm \frac{1}{2}} \varphi\left(p_{ \pm}, s_{3}\right) e\left(\dot{p}_{ \pm}, s_{3}\right) .
\end{gathered}
$$

Thus the field representation is equivalent to the direct sum

$$
\left[+m, \frac{1}{2}\right] \oplus\left[-m, \frac{1}{2}\right] .
$$

$$
e\left(\dot{p}_{+},+\frac{1}{2}\right)=\frac{1}{\sqrt{2}}\left(\begin{array}{l}
1 \\
0 \\
1 \\
0
\end{array}\right), \quad e\left(\dot{p}_{+},-\frac{1}{2}\right)=\frac{1}{\sqrt{2}}\left(\begin{array}{r}
0 \\
1 \\
0 \\
-1
\end{array}\right), \quad e\left(\dot{p}_{-},+\frac{1}{2}\right)=\frac{1}{\sqrt{2}}\left(\begin{array}{l}
0 \\
1 \\
0 \\
1
\end{array}\right), \quad e\left(\dot{p}_{-},-\frac{1}{2}\right)=\frac{1}{\sqrt{2}}\left(\begin{array}{r}
1 \\
0 \\
-1 \\
0
\end{array}\right) .
$$

Under an element of the stationary subgroup they transform according to

$$
D\left(\dot{\mathcal{A}}_{ \pm}\right) e\left(\dot{p}_{ \pm}, s_{3}\right)=e^{i s_{3} a_{ \pm}} e\left(\dot{p}_{ \pm}, s_{3}\right) .
$$

To sum up:

$$
\begin{aligned}
U(\mathrm{a}, \mathcal{A}) \varphi\left(p_{ \pm}, s_{3}\right) e^{i \mathrm{a} p} & =\exp \left\{i s_{3} \alpha(\mathcal{A}, p \pm)\right\} \varphi\left(\Lambda^{-1} p_{ \pm}, s_{3}\right)
\end{aligned}
$$

$$
C=\frac{1}{\sqrt{2}}\left(\begin{array}{rr}
1 & +1 \\
-1 & 1
\end{array}\right), \quad D(\mathcal{A})=C^{-1} A C .
$$

In this representation $\gamma^{0}$ is diagonal:

$$
\gamma^{0}=\left(\begin{array}{rr}
\mathbf{1} & 0 \\
0 & -\mathbf{1}
\end{array}\right), \quad \gamma^{k}=\left(\begin{array}{cc}
0 & \sigma^{k} \\
-\sigma^{k} & 0
\end{array}\right) .
$$

$m>0$ :

The Dirac equation in $p$-space admits the following solutions for $p_{ \pm}=\dot{p}_{ \pm}$:

It can easily be shown that (4.8) indeed leads to the well known solutions of the Dirac-equation.

The scalar product takes the usual form:

$$
\left\langle\psi\left(p_{ \pm}\right), \psi^{\prime}\left(p_{ \pm}\right)\right\rangle= \pm \psi^{\dagger}\left(p_{ \pm}\right) \gamma^{0} \psi^{\prime}\left(p_{ \pm}\right) \text {. }
$$

Finally, it is readily proved that with the help of the discrete transformations the representation gets a unitary and irreducible representation of the full Poincaré group.

$m=0$ :

The basic solutions for $p_{ \pm}=\dot{p}_{ \pm}$are:

where

$$
\varphi\left(p_{ \pm}\right)=\sum_{s_{3}= \pm \frac{1}{2}} \varphi\left(p_{ \pm}, s_{3}\right) e\left(\dot{p}_{ \pm}, s_{3}\right) .
$$

Hence the field representation is equivalent to the direct sum

$\left[+0,+\frac{1}{2}\right] \oplus\left[+0,-\frac{1}{2}\right] \oplus\left[-0,+\frac{1}{2}\right] \oplus\left[-0,-\frac{1}{2}\right]$.

With the help of

$$
D\left(A_{p_{ \pm}}\right)=\frac{1}{\sqrt{2\left(\omega+p^{3}\right)}}\left(\begin{array}{cccc}
1+\omega & 0 & \mp\left(1-p^{3}\right) & \pm p^{-} \\
0 & 1+\omega & \pm p^{+} & \pm\left(1-p^{3}\right) \\
\mp\left(1-p^{3}\right) & \pm p^{-} & 1+\omega & 0 \\
\pm p^{+} & \pm\left(1-p^{3}\right) & 0 & 1+\omega
\end{array}\right), \quad p^{ \pm}=p^{1} \pm i p^{2},
$$


we get the following basis:

$$
\begin{aligned}
& e\left(p_{+},+\frac{1}{2}\right)=\frac{1}{2 \sqrt{\omega+p^{3}}}\left(\begin{array}{c}
\omega+p^{3} \\
p^{+} \\
\omega+p^{3} \\
p^{+}
\end{array}\right), \quad e\left(p_{+},-\frac{1}{2}\right)=\frac{1}{2 \sqrt{\omega+p^{3}}}\left(\begin{array}{c}
-p^{-} \\
\omega+p^{3} \\
p^{-} \\
-\omega-p^{3}
\end{array}\right), \\
& e\left(p_{-},+\frac{1}{2}\right)=\frac{1}{2 \sqrt{\omega+p^{3}}}\left(\begin{array}{c}
-p^{-} \\
\omega+p^{3} \\
-p^{-} \\
\omega+p^{3}
\end{array}\right), \quad e\left(p_{-},-\frac{1}{2}\right)=\frac{1}{2 \sqrt{\omega+p^{3}}}\left(\begin{array}{c}
\omega+p^{3} \\
p^{+} \\
-\omega-p^{3} \\
-p^{+}
\end{array}\right) .
\end{aligned}
$$

The scalar product (4.10) can be transformed into:

$$
\left\langle\psi\left(p_{ \pm}\right), \psi^{\prime}\left(p_{ \pm}\right)\right\rangle=\frac{1}{\omega} \psi^{\dagger}\left(p_{ \pm}\right) \psi^{\prime}\left(p_{ \pm}\right) .
$$

The investigation of the discrete transformations proceeds as follows: One can show that $T\left(\mathcal{A}_{\mathrm{S}}\right) \psi\left(\boldsymbol{p}_{ \pm}\right)$ $=\gamma^{0} \psi\left(\Lambda_{\mathrm{S}} p_{ \pm}\right)$is a solution of the Dirac-equation with fixed point $\dot{p}^{\prime}{ }_{ \pm}=( \pm 1,0,0,-1)$. This fixed point is contained in the orbit through $\dot{p}_{ \pm}$. The Wigner boost $\mathcal{A}_{p_{ \pm}}^{\prime}$ for the fixed point $\dot{p}^{\prime}{ }_{ \pm}$is related to (2.24) by the equation

where

$$
\mathcal{A}_{p_{ \pm}}^{\prime}=\mathcal{A}_{\Lambda_{s} p_{ \pm}}^{-1}=\mathcal{A}_{p_{ \pm}} \dot{\mathcal{A}}_{p_{ \pm}} \varepsilon^{-1}
$$

and

$$
\varepsilon=\left(\begin{array}{rr}
0 & 1 \\
-1 & 0
\end{array}\right), \quad \dot{A}_{p+}=\left(\begin{array}{cc}
e^{+i \chi_{+} / 2} e^{-i \chi_{+} / 2} z_{+} \\
0 & e^{-i \chi_{+}+2}
\end{array}\right), \quad \dot{A}_{p-}=\left(\begin{array}{ll}
e^{-i \chi_{-} / 2} & 0 \\
e^{-i \chi_{-} / 2} z_{-} & e^{+i \chi_{-} / 2}
\end{array}\right)
$$

$$
\begin{gathered}
e^{+i_{+} / 2}=-p^{1}+i p^{2} / \sqrt{\left(\omega+p^{3}\right)\left(\omega-p^{3}\right)}=e^{-i_{\chi_{-} / 2}}, \\
z_{+}=-2(1+\omega) p^{-} /\left(\omega+p^{3}\right)\left(\omega-p^{3}\right)=z_{-}{ }^{*}, z_{ \pm}=z_{ \pm}{ }^{1}+i z_{ \pm}{ }^{2} .
\end{gathered}
$$

This yields

$$
\begin{aligned}
& T\left(\mathcal{A}_{\mathrm{S}}\right) e\left(p_{+}, \pm \frac{1}{2}\right)= \pm e^{\mp i_{\chi_{+} / 2}} e\left(p_{+}, \mp \frac{1}{2}\right) \\
& T\left(\mathcal{A}_{\mathrm{S}}\right) e\left(p_{-}, \pm \frac{1}{2}\right)=\mp e^{\mp i_{-} / 2} e\left(p_{-}, \mp \frac{1}{2}\right)
\end{aligned}
$$

The action of the time inversion is:

$$
\begin{aligned}
& T\left(\mathcal{A}_{\mathrm{T}}\right) e\left(p_{+}, \pm \frac{1}{2}\right)= \pm e\left(p_{-}, \mp \frac{1}{2}\right), \\
& T\left(\mathcal{A}_{\mathrm{T}}\right) e\left(p_{-}, \pm \frac{1}{2}\right)= \pm e\left(p_{+}, \mp \frac{1}{2}\right) .
\end{aligned}
$$

This representation, however, is equivalent to the corresponding representation of $(2.30)$. $s=1 ; m>0$ :

The equivalent representation (4.4)

$$
[U(\mathrm{a}, \mathcal{A}, \Lambda) \varphi]^{\nu}\left(p_{ \pm}\right)=e^{i \mathrm{a} p_{ \pm}}\left(\Lambda_{p_{ \pm}}^{-1} \Lambda \Lambda_{\Lambda^{-1} p_{ \pm}}\right)^{\nu}{ }_{\mu} \varphi^{\mu}\left(\Lambda^{-1} p_{ \pm}\right)
$$

decomposes into the direct sum $[ \pm m, 0] \oplus[ \pm m, 1]$. The Lorentz condition requires however $\varphi^{0}\left(p_{ \pm}\right)=0$; this annihilates the term with spin 0 .

The basis is formed with the help of $e_{k}^{\mu}\left(\dot{p}_{ \pm}\right)=g_{k}^{\mu}$ :

The canonical basis is

$$
e_{k}\left(p_{ \pm}\right)=\Lambda_{p_{ \pm}} e_{k}\left(\dot{p}_{ \pm}\right)=\left(\begin{array}{l}
\frac{\mp p_{k}}{m} \\
g_{k}^{i}-\frac{p^{i} p_{k}}{m(\omega+m)}
\end{array}\right)_{i=1,2,3}
$$

$$
\begin{aligned}
& e\left(p_{+}, \pm 1\right)=\frac{1}{\sqrt{2}}\left[e_{1}\left(p_{+}\right) \pm i e_{2}\left(p_{+}\right)\right], \\
& e\left(p_{-}, \pm 1\right)=\frac{1}{\sqrt{2}}\left[e_{1}\left(p_{-}\right) \pm i e_{2}\left(p_{-}\right)\right],
\end{aligned}
$$


Using (4.10), we get for the scalar product:

$$
\left\langle\psi\left(p_{ \pm}\right), \psi^{\prime}\left(p_{ \pm}\right)\right\rangle=-\psi_{\mu}^{*}\left(p_{ \pm}\right) \psi^{\prime \mu}\left(p_{ \pm}\right) .
$$

Because of

$$
\begin{gathered}
T\left(\Lambda_{\mathrm{S}}\right) e\left(p_{ \pm}, s_{3}\right)=-e\left(p_{ \pm}, s_{3}\right), \\
T\left(\Lambda_{\mathrm{T}}\right) e\left(p_{ \pm}, s_{3}\right)=e\left(p_{\mp}, s_{3}\right),
\end{gathered}
$$

the equivalence to a unitary and irreducible representation (2.29) of $\tilde{P}$ is readily verified.

$m=0$ :

The Lorentz condition permits the solutions

$$
e\left(\dot{p}_{+}, \pm 1\right)=\frac{1}{\sqrt{2}}\left(\begin{array}{c}
0 \\
1 \\
\pm i \\
0
\end{array}\right)=e\left(\dot{p}_{-}, \mp 1\right), \quad e\left(\dot{p}_{ \pm}, 0\right)=\frac{1}{\sqrt{2}}\left(\begin{array}{r} 
\pm 1 \\
0 \\
0 \\
1
\end{array}\right) .
$$

An element of the stationary subgroup transforms them as follows:

$$
\begin{gathered}
\dot{\Lambda}_{ \pm} e\left(\dot{p}_{ \pm}, s_{3}\right)=e^{i s_{3} x_{ \pm}} e\left(\dot{p}_{ \pm}, s_{3}\right) \pm e^{i s_{3} x_{ \pm}}\left(u_{ \pm}-i s_{3} v_{ \pm}\right) e\left(\dot{p}_{ \pm}, 0\right), \\
\dot{\Lambda}_{ \pm} e\left(\dot{p}_{ \pm}, 0\right)=e\left(\dot{p}_{ \pm}, 0\right), \quad s_{3}= \pm 1 .
\end{gathered}
$$

This representation of $E(2)$ is reducible, but not decomposable: The multiples of $e\left(\dot{p}_{ \pm}, 0\right)$ form an invariant subspace; however, an equivalent representation leaving invariant the subspace spanned e. $g$. by $e\left(\dot{p}_{ \pm},+1\right)$ does not exist. The computation of the basis with the help of (2.25) yields:

where

$$
\begin{aligned}
& e\left(p_{+}, \pm 1\right)=\hat{e}\left(p_{+}, \pm 1\right)+\frac{p^{ \pm}}{\omega+p^{3}} e\left(p_{+}, 0\right), \\
& e\left(p_{-}, \pm 1\right)=\hat{e}\left(p_{-}, \pm 1\right)+\frac{p^{\mp}}{\omega+p^{3}} e\left(p_{-}, 0\right),
\end{aligned}
$$

$$
\hat{e}\left(p_{+}, \pm 1\right)=\frac{1}{\sqrt{2}}\left(\begin{array}{c}
p^{ \pm} / \omega+p^{3} \\
1 \\
\pm i \\
-p^{ \pm} / \omega+p^{3}
\end{array}\right), \quad \hat{e}\left(p_{-}, \pm 1\right)=\frac{1}{\sqrt{2}}\left(\begin{array}{c}
-p^{\mp} / \omega+p^{3} \\
\frac{1}{\mp i} \\
-p^{\mp} / \omega+p^{3}
\end{array}\right) \text {. }
$$

An element of the identity component acts on the basis elements with $s_{3}= \pm 1$ according to

$$
\begin{aligned}
T(\mathrm{a}, \Lambda)\left\{\varphi\left(p_{ \pm}, s_{3}\right) e\left(p_{ \pm}, s_{3}\right)\right\} & =e^{i \mathrm{a} p_{ \pm}} e^{i s_{3} \alpha\left(\Lambda, p_{ \pm}\right)} \varphi\left(\Lambda^{-1} p_{ \pm}, s_{3}\right) e\left(p_{ \pm}, s_{3}\right) \\
& \pm e^{i \mathrm{a} p_{ \pm}} e^{i s_{3} \alpha\left(\Lambda, p_{ \pm}\right)} \varphi\left(\Lambda^{-1} p_{ \pm}, s_{3}\right)\left[u\left(\Lambda, p_{ \pm}\right)-i s_{3} v\left(\Lambda, p_{ \pm}\right)\right] e\left(p_{ \pm}, 0\right) .
\end{aligned}
$$

By means of (4.20) and (4.21) we get

$$
T\left(\Lambda_{\mathrm{S}}\right) e\left(p_{ \pm}, s_{3}\right)=e^{-i s_{3} \gamma_{ \pm}} e\left(p_{ \pm},-s_{3}\right) \pm e^{-i s_{3} \chi_{ \pm}}\left(z_{ \pm}^{1}+i s_{3} z_{ \pm}^{2}\right) e\left(p_{ \pm}, 0\right)
$$

for the effect of space inversion and

$$
T\left(\Lambda_{\mathrm{T}}\right) e\left(p_{ \pm}, s_{3}\right)=e\left(p_{\mp},-s_{3}\right)
$$

for the time inversion.

To achieve irreducibility of this representation, we compute the scalar product for the (non-invariant) subspace of functions $\psi\left(p_{ \pm}\right)$with helicity $s_{3}=+1$ and $s_{3}=-1$ which it inherits from the corresponding Wignerian representation.

As to be expected, the result is:

$$
\left\langle\psi\left(p_{ \pm}\right), \psi^{\prime}\left(p_{ \pm}\right)\right\rangle=-\psi_{\mu}^{*}\left(p_{ \pm}\right) \psi^{\prime \mu}\left(p_{ \pm}\right) .
$$

If we use this scalar product for the whole space we obtain:

$$
\left\langle e\left(p_{ \pm}, s_{3}\right), e\left(p_{ \pm}, s_{3}{ }^{\prime}\right)\right\rangle=\delta_{s_{3} s_{3}},\left\langle e\left(p_{ \pm}, 0\right), e\left(p_{ \pm}, 0\right)\right\rangle=0,\left\langle e\left(p_{ \pm}, s_{3}\right), e\left(p_{ \pm}, 0\right)\right\rangle=0 .
$$


The functions of the form $\varphi\left(p_{ \pm}, 0\right) e\left(p_{ \pm}, 0\right)$ thus have norm 0 and are orthogonal to

$$
\psi\left(p_{ \pm}\right)=\sum_{s_{3}= \pm 1} \varphi\left(p_{ \pm}, s_{3}\right) e\left(p_{ \pm}, s_{3}\right) .
$$

For this reason we introduce the following equivalence relation ${ }^{18}: \psi^{\prime}\left(p_{ \pm}\right)$is defined to be equivalent to $\psi\left(p_{ \pm}\right)$if

$$
\psi^{\prime}\left(p_{ \pm}\right)=\psi\left(p_{ \pm}\right)+\lambda\left(p_{ \pm}\right) p_{ \pm} .
$$

The representative of the class of $\psi\left(p_{ \pm}\right)$we denote by $\hat{\psi}\left(p_{ \pm}\right)$[see (4.31)]. On the quotient space the representation is unitary and irreducible as a representation of $\tilde{P}$ :

$$
\begin{gathered}
T(\mathrm{a}, \Lambda)\left\{\varphi\left(p_{ \pm}, s_{3}\right) \hat{e}\left(p_{ \pm}, s_{3}\right)\right\}=e^{i a p_{ \pm}} e^{i s_{3} a\left(\Lambda, p_{ \pm}\right)} \varphi\left(\Lambda^{-1} p_{ \pm}, s_{3}\right) \hat{e}\left(p_{ \pm}, s_{3}\right) \\
T\left(\Lambda_{\mathrm{S}}\right) \hat{e}\left(p_{ \pm}, s_{3}\right)=e^{-i s_{3} \chi_{ \pm}} \hat{e}\left(p_{ \pm},-s_{3}\right), \quad T\left(\Lambda_{\mathrm{T}}\right) \hat{e}\left(p_{ \pm}, s_{3}\right)=\hat{e}\left(p_{\mp},-s_{3}\right)
\end{gathered}
$$

where $\left.\quad \alpha\left(\Lambda, p_{ \pm}\right)=\arccos \left(\Lambda_{p_{ \pm}}^{-1} \Lambda \Lambda_{\Lambda^{-1} p_{ \pm}}\right)_{1}{ }_{1}, e^{+i_{\chi_{+} / 2}}=-p^{1}+i p^{2} \overline{\sqrt{\left(\omega+p^{3}\right.}}\right)\left(\overline{\left.-p^{3}\right)}=e^{-i_{\chi_{-} / 2}}\right.$.

The basis (4.31) belongs to the Lorentz gauge. In concluding we add the corresponding results for the Coulomb gauge which is obtained in the helicity formalism. The computation of the basis yields:

$$
\hat{e}\left(p_{+}, \pm 1\right)=\frac{1}{\sqrt{2}}\left(\begin{array}{c}
\frac{p^{3}}{\omega} \mp i \frac{p^{2}}{\omega\left(\omega+p^{3}\right)} p^{ \pm} \\
\frac{p^{3}}{\omega} \mp i \frac{p^{1}}{\omega\left(\omega+p^{3}\right)} p^{ \pm} \\
-\frac{p^{ \pm}}{\omega}
\end{array}\right)=\hat{e}\left(p_{-}, \mp 1\right) \text {. }
$$

Indeed, these solutions satisfy $\hat{e}^{0}\left(p_{ \pm}, s_{3}\right)=0$. They transform in exactly the same way as has been given in (4.37).

\section{Acknowledgement}

It is a pleasure to thank Dr. L. Wiedecke for stimulating discussions. This work was supported in part by the Deutsche Forschungsgemeinschaft.

1 W. Gordon, Z. Phys. 40, 117 [1926].

2 O. Klein, Z. Phys. 37, 895 [1926].

3 P. A. M. Dirac, Proc. Roy. Soc, A 117, 610 [1928].

4 M. Fierz, Helv. Phys. Acta 12, 3 [1939].

5 W. Rarita and J. Schwinger, Phys. Rev. 60, 61 [1941].

6 V. Bargmann and E. P. Wigner, Proc. Nat. Acad. Sci. U.S.A. 34, 211 [1948].

7 H. Joos, Fortschr. Phys. 10, 65 [1962].

${ }^{8}$ I. M. Gelfand, R. A. Minlos, and Z. Ya. Shapiro, Representations of the Rotation and Lorentz Groups and Their Applications, Pergamon Press, London 1963.

9 M. A. Neumark, Lineare Darstellungen der Lorentzgruppe. Berlin, VEB Deutscher Verlag der Wissenschaften 1963.

10 A. Salam, R. Delbourgo, and J. Strathdee, Proc. Roy. Soc. London A 284, 146 [1965].

11 D. L. Pursey, Ann. Phys. 32, 157 [1965].

12 This work forms part of the authors dissertation, Kiel, University of Kiel 1971.

13 G. W. Mackey, Group Representations and Non-Commutative Harmonic Analysis, Univ. of California Press, Berkeley 1965.
14 R. Herman, Lie Groups for Physicists, W. A. Benjamin, New York 1966.

15 R. Herman, Vector Bundles in Mathematical Physics, Vol. I, II, W. A. Benjamin, New York 1970.

16 G. W. Mackey, The Theory of Group Representations, Univ. of Chicago 1955.

17 E. P. Wigner, Ann. Math. 40, 149 [1939].

18 A. S. Wightman, in C. de Witt and R. Omnes, eds., Relations de dispersion et particules élémentaires. Hermann, Paris 1960.

19 Ideas related to those which we present here have been expressed by R. Herman in ${ }^{14}$; he comes close to ours in ${ }^{15}$.

20 G. Grensing, in preparation.

21 M. Jacob and G. C. Wick, Ann. Phys. 7, 404 [1959].

22 Yu. M. Shirokov, Sov. Phys. JETP 34, 493 [1958]; 36, 620 [1959].

23 A. H. Clifford, Ann. Math. 38, 533 [1937].

24 E. P. Wigner, in F. Gursey, ed., Group Theoretical Concepts and Methods in Elementary Particle Physics, Gordon and Breach, New York-London 1964.

25 A. J. Coleman, in M. Loebl, ed., Group Theory and Its Applications, Academic Press, New York-London 1968. 